\title{
Resangrado de la úlcera péptica en pacientes con y sin enfermedad renal crónica
}

\author{
Gerly Edson Guzmán Calderón 1,2,3,a,b
}

RESUMEN

Objetivo: Determinar si el resangrado de la enfermedad ulcerosa péptica (EUP) en pacientes con enfermedad renal crónica (ERC) es mayor que en los pacientes con EUP sin ERC en los pacientes hospitalizados en la Unidad de Hemorragia Digestiva del Hospital Nacional Edgardo Rebagliati Martins.

Materiales y métodos: Se realizó un estudio de casos y controles, observacional, analítico y retrosprospectivo en pacientes mayores de 18 años, con resangrado por úlcera péptica, ingresados a la Unidad de Hemorragia Digestiva del Hospital Nacional Edgardo Rebagliati Martins durante un periodo de 1 año.

Resultados: Total de 146 pacientes, 26 \% son mujeres y $74 \%$ varones. Media de edad: 65,47 $\pm 17,95$ años. Los casos (EUP con ERC) fueron 34 y los controles (EUP sin ERC) fueron 112. La causa del sangrado fue úlcera duodenal en $53 \%$ y úlcera gástrica en $47 \%$. La razón entre la presencia de resangrado en pacientes con EUP con ERC es 6,43 veces mayor que en pacientes con EUP sin ERC. Resangrado en pacientes con ERC 41 , 1 \% y en los pacientes sin ERC de 9,8\%. El tipo de úlcera con mayor resangrado fue el tipo lla en ambos grupos. El $94 \%$ de pacientes fueron dados de alta, 2,7 \% referidos a cirugía. La tasa de mortalidad total fue de 2,7 \%, y de 5,8 \% en los pacientes con ERC. $(p=0,2)$.

Conclusiones: La razón de resangrado de la úlcera péptica en pacientes que padecen de enfermedad renal crónica es 6,43 veces mayor que en los pacientes sin enfermedad renal crónica, La tasa de resangrado de la enfermedad ulcerosa péptica en pacientes con enfermedad renal crónica, fue del $41,1 \%$, mientras que en los pacientes sin enfermedad renal crónica fue de 9,8\%.

Palabras clave: Ulcera péptica; Enfermedad renal crónica; Complicaciones (Fuente: DeCS BIREME).

\section{Peptic ulcer rebleeding in patients with and without chronic kidney disease}

\author{
ABSTRACT
}

Objective: To determine if rebleeding in peptic ulcer disease (PUD) in patients with chronic kidney disease (CKD) is greater than in patients without CKD hospitalized in the Digestive Bleeding Unit of the Hospital Nacional Edgardo Rebagliati Martins.

Materials and methods: An observational, analytical, retro-prospective, case-control study was performed in patients $>18$ years with rebleeding in PUD, admitted to the Digestive Hemorrhage Unit of the Hospital Nacional Edgardo Rebagliati Martins during 1 year.

Results: Out of 146 patients, $26 \%$ were female and $74 \%$ were male. The mean age was $65.47 \pm 17.95$ years. The cases (PUD with CKD) consisted of 34 patients and the controls (PUD without CKD) consisted of 112 patients. The causes of rebleeding were duodenal ulcer $(53 \%)$ and gastric ulcer $(47 \%)$. The odds ratio (OR) of rebleeding in patients with CKD was 6.43 times higher than in patients without CKD. Rebleeding in patients with CKD was $41.1 \%$ and in patients without CKD it was $9.8 \%$. The type of ulcer with the greatest rebleeding was type lla in both groups. Ninety-four percent (94\%) of the patients were discharged, and $2.7 \%$ were referred to surgery. The total mortality rate was $2.7 \%$, and the mortality rate in patients with CKD was $5.8 \%(p=0.2)$.

Conclusions: The OR of peptic ulcer rebleeding in patients with CKD is 6.43 times higher than in patients without CKD. Rebleeding in peptic ulcer disease in patients with CKD was $41.1 \%$, whereas in patients without CKD it was $9.8 \%$.

Keywords: Peptic ulcer; Chronic kidney disease; Complications (Source: MeSH NLM).

1. Hospital Nacional Edgardo Rebagliati Martins. Lima, Perú.

2. Clínica Internacional. Lima, Perú.

3. Universidad Peruana de Ciencias Aplicadas. Lima, Perú.

a. Gastroenterólogo.

b. Profesor de la Escuela de Medicina. 


\section{INTRODUCCIÓN}

La enfermedad ulcerosa péptica (EUP) es una de las emergencias médicas más comunes la cual contribuye a una significativa morbilidad y mortalidad en todo el mundo, se menciona que incluso el 5 al $10 \%$ de la población presentará alguna manifestación de enfermedad ulcerosa péptica a lo largo de su vida ${ }^{(1)}$. Por otra parte, la enfermedad renal crónica (ERC) es otro cuadro de alta prevalencia en nuestro medio, y una de las patologías que resulta ser una complicación de enfermedades como la hipertensión arterial o la diabetes mellitus, es la causa más frecuente de hospitalización en los servicios de nefrología a nivel nacional (2).

Algunos estudios refieren que la enfermedad renal crónica es una patología que incrementa el riesgo de sangrado en los pacientes con úlcera péptica, que es la mayor causa de hemorragia digestiva en los pacientes con enfermedad renal crónica. Se ha descrito que los pacientes con enfermedad renal crónica tienen mayor riesgo de resangrado de la úlcera péptica, lo cual ocasionaría mayor cantidad de complicaciones en los pacientes nefrópatas. La patogénesis de la hemorragia digestiva en pacientes con enfermedad ulcerosa péptica y enfermedad renal crónica aún no es muy clara, sin embargo, se han sugerido algunas hipótesis que podrían explicar esta mayor incidencia de complicaciones en los pacientes nefrópatas: la disfunción plaquetaria de origen urémico, que se cree que es el factor más importante, la acidez y la integridad de la mucosa gástrica, las cuales están alteradas en los pacientes con enfermedad renal crónica (3).

El objetivo de la presente revisión es dar a conocer la tasa de complicaciones de los pacientes con enfermedad renal crónica, específicamente, el resangrado, las complicaciones que requieran tratamiento quirúrgico y finalmente, la mortalidad en este grupo de pacientes.

\section{MATERIALES Y MÉTODOS}

Es un estudio de tipo descriptivo, retroprospectivo y transversal. Se estudiaron los pacientes de la Unidad de Hemorragia Digestiva del Hospital Nacional Edgardo Rebagliati Martins con diagnóstico de enfermedad ulcerosa péptica y enfermedad renal crónica que fueron hospitalizados en un periodo de 1 año. Se recolectaron datos de libro de ingresos de la Unidad de Hemorragia Digestiva. Entre los criterios de inclusión se tomaron en cuenta a pacientes mayores de 18 años, hospitalizados en la Unidad de Hemorragia Digestiva cuya causa de hospitalización fue enfermedad ulcerosa péptica (gástricas y/o duodenales), y que tengan como antecedente padecer cualquier grado de enfermedad renal crónica, los pacientes debían de contar con una endoscopía digestiva alta con el respectivo consentimiento informado. Se excluyeron a los pacientes menores de 18 años, o aquellos cuya causa de la hemorragia digestiva fuera diferente de una enfermedad ulcerosa péptica como por ejemplo, síndrome de Mallory Weiss, angiodisplasias, hemorragia por várices esofágicas y/o gástricas, lesión de Dieulafoy o cáncer gástrico, esofágico o duodenal. Además, se excluyeron pacientes sin consentimiento informado para endoscopía o con datos incompletos.

Los pacientes fueron seleccionados de acuerdo al diagnóstico de ingreso cuando llegaron a la Unidad de Hemorragia Digestiva del Hospital Nacional Edgardo Rebagliati Martins, el dato de si el paciente padecía de enfermedad renal crónica fue tomado tanto directamente de la nota de ingreso proveniente de la historia clínica o del libro de registro de ingresos de la Unidad de Hemorragia Digestiva. El estadiaje de la enfermedad renal crónica, se hizo de acuerdo al KDIGO (Kidney Disease: Improving Global Outcomes)(4,5). Se procedió a la toma de datos demográficos y a llenarlos en la ficha de recolección de datos. Los pacientes fueron divididos inicialmente en 2 grupos de estudio. Los grupos de estudio corresponden a todos aquellos pacientes con enfermedad ulcerosa péptica portadores de enfermedad renal crónica y el grupo control, pacientes con enfermedad ulcerosa péptica sin enfermedad renal crónica. Finalmente, se analizaron comparativamente los datos demográficos, laboratoriales, así como la presencia de comorbilidades concomitantes como hipertensión arterial, diabetes mellitus, cardiopatías o consumo de antiinflamatorios no esteroideos (AINE), para finalmente comparar las complicaciones (resangrado fundamentalmente), entre los dos grupos de estudio.

El tratamiento realizado en ambos grupos fue similar, de acuerdo a los protocolos de manejo internacionales de hemorragia digestiva alta no varicosa, es decir, un inhibidor de bomba de protones (omeprazol), $80 \mathrm{mg}$ VEV en bolo y después $40 \mathrm{mg}$ VEV cada 12 horas. Posteriormente, el tratamiento de mantenimiento fue por vía oral dependiendo del inicio de la tolerancia oral. La terapia de mantenimiento fue por 8 a 12 semana para úlceras gástricas y de 4 a 8 semanas para la úlcera duodenal. El tratamiento endoscópico fue similar para cada grupo y dependió de la clasificación de Forrest. Los pacientes con Forrest la, lb, Ila y llb, fueron sometidos a terapia endoscópica doble (inyectoterapia con adrenalina, más un método mecánico o térmico), dependiendo de la elección y experiencia del endoscopista. El tiempo de ingreso y el inicio del tratamiento endoscópico fue variable, pero casi siempre dentro de las 72 horas del ingreso.

\section{Análisis estadístico}

Estadística descriptiva: Se determinó la media y desviación estándar de los diferentes datos demográficos del paciente. Estadística inferencial: Para evaluar la diferencia de medias entre los grupos (casos y controles) se 
utilizó la prueba t de Student o Kruskal-Wallis. Para datos cualitativos o categóricos se evaluaron las diferencias entre los grupos (casos y controles) mediante la prueba chi cuadrado. Se realizó el análisis del Odds Ratio (OR) para determinar la repercusión de la enfermedad renal crónica en el resangrado de la úlcera péptica. Se utilizó el paquete estadístico SPSS 20 para Windows. Se consideró en cada caso el intervalo de confianza al $95 \%$ y el respectivo criterio de significancia (valor de $\mathrm{p}<0.05$ ) como estadísticamente significativo.

\section{RESULTADOS}

En el presente estudio se evaluaron un total de 146 pacientes. El $100 \%$ de los pacientes fueron casos admitidos a la Unidad de Hemorragia Digestiva del Hospital Nacional Edgardo Rebagliati Martins por un diagnóstico de hemorragia digestiva alta que tuvo como etiología la enfermedad ulcerosa péptica.

En la tabla 1, podemos apreciar los datos demográficos de los pacientes incluidos en el estudio. De los 146 pacientes, $26 \%$ son de sexo femenino y el $74 \%$ son de sexo masculino. La media y la desviación estándar de las edades del total de pacientes fue de 65,47 \pm 17,95 años. El rango de edades de los pacientes osciló entre los $19 \mathrm{y}$ 93 años.

Tabla 1. Datos demográficos de los pacientes en estudio

\begin{tabular}{lc} 
& Datos demográficos generales \\
\hline Edad & $65,5 \pm 17,9$ años \\
Sexo: (varón/mujer) & $108 / 38$ \\
Ubicación: gástrica/duodenal $(\mathrm{n}=146)$ & $68 / 78$ \\
Comorbilidad (Sí/No) & $76 / 70$ \\
Tasa de complicaciones & $21,2 \%$ \\
Tasa de mortalidad & $2,7 \%$ \\
\hline
\end{tabular}

Los dos grupos de estudio fueron analizados de acuerdo al perfil demográfico, como se observa en la tabla 2. Si bien es cierto que los pacientes con enfermedad ulcerosa péptica con enfermedad renal crónica tuvieron un promedio de edad algo mayor que los pacientes sin enfermedad renal crónica, creemos que esta diferencia no resulta sustancial a la hora de analizar las tasas de resangrado. El conteo de plaquetas estuvo, en promedio, dentro de los valores normales en ambos grupos de estudio; aunque los pacientes con enfermedad renal crónica tuvieron un conteo algo más bajo que el del grupo sin enfermedad renal crónica. Sin embargo, existe la posibilidad de que la funcionalidad plaquetaria se vea alterada en el grupo con nefropatía, y esto conllevar a una mayor tasa de resangrado, pero esto no fue analizado en nuestro estudio.

Tabla 2. Diferencias demográficas entre los 2 grupos de estudio

\begin{tabular}{|c|c|c|c|c|}
\hline & $\begin{array}{c}\text { EUP } \\
(n=112)\end{array}$ & $\begin{array}{c}\text { EUP + ERC } \\
(n=34)\end{array}$ & $\mathbf{p}$ & Significancia \\
\hline Edad $\left(X^{-} \pm S D\right)$ & $63,7 \pm 18,4$ & $72,1 \pm 14,5$ & 0,01 & Sí \\
\hline Sexo masculino (\%) & $80(71 \%)$ & $28(82 \%)$ & 0,1 & NS \\
\hline Hemoglobina (g/dl) & $8,8 \pm 2,4$ & $7,5 \pm 3,0$ & 0,009 & Sí \\
\hline Plaquetas (X 109 /L) & $238 \pm 86$ & $188 \pm 93$ & 0,004 & Sí \\
\hline Creatinina (mg/dL) & $0,8 \pm 0,3$ & $5,3 \pm 3,1$ & $<0,001$ & Sí \\
\hline Tiempo protombina (s) & $12,9 \pm 2,0$ & $12,5 \pm 1,2$ & 0,3 & NS \\
\hline AINE n (\%) & $22(19 \%)$ & $4(12 \%)$ & 0,2 & NS \\
\hline
\end{tabular}

Abreviaturas: EUP: Enfermedad ulcerosa péptica. ERC: Enfermedad renal crónica. EUP+ERC: Enfermedad ulcerosa péptica con enfermedad renal crónica. AINE : Antiinflamatorios no esteroideos. 
Cuando se clasificaron las úlceras pépticas de acuerdo a la clasificación de Forrest, se pudo observar que la mayoría de los pacientes con sangrado digestivo con este origen estaban en un estadio Ila, $(39,7 \%)$, seguido de las úlceras en estadio Forrest IIc, (26\%). De los pacientes con úlcera gástrica, el estadio lla estuvo presente en el $44,1 \%$.
No se observaron pacientes con Forrest la. En los pacientes con úlceras duodenales, la mayoría de los pacientes con sangrado digestivo también estaban en un estadio lla, $(35,9 \%)$, mientras que en este grupo si hubo 4 pacientes con Forrest la (Figura 1).

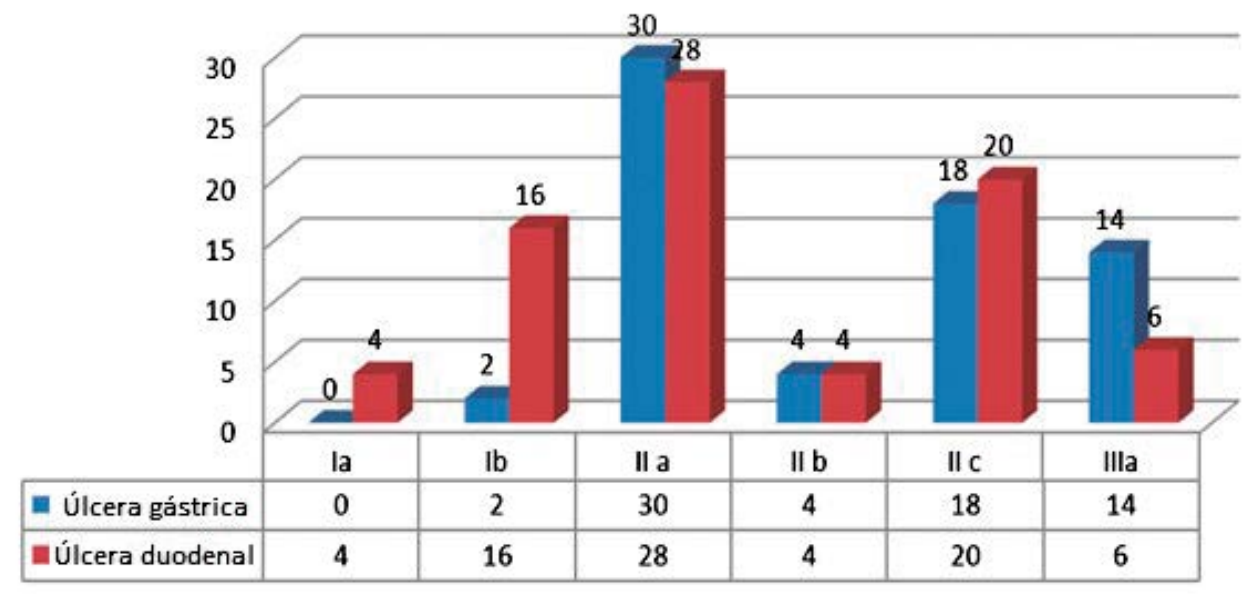

Figura 1. Distribución de las úlceras pépticas de acuerdo a la clasificación de Forrest

Cuando se hizo el análisis de resangrado de acuerdo a la clasificación de Forrest apreciamos que la mayor tasa de resangrados se vio en pacientes con Forrest Ilb. Es probable que esto se explique por el bajo número de casos que se presentaron con esta variante. Proporcionalmente, las úlceras con sangrado activo o mayores estigmas de sangrado, tuvieron una recurrencia de entre $17 \%$ y $27 \%$.
El resangrado en los grupos de bajo riesgo (Ilc y III) estuvieron alrededor del 10 \% (Tabla 3). Vale la pena mencionar que todos los pacientes con Forrest la, Ib, lla y llb, fueron sometidos a tratamientos endoscópicos combinados de acuerdo a las guías de manejo internacionales de hemorragia digestiva.

Tabla 3. Tasa de resangrado de acuerdo a la clasificación de Forrest

\begin{tabular}{cccc|}
$\begin{array}{c}\text { Clasificación de } \\
\text { Forrest }\end{array}$ & Número & $\begin{array}{c}\text { Número de } \\
\text { resangrados }\end{array}$ & $\begin{array}{c}\text { Tasa de } \\
\text { resangrado }\end{array}$ \\
\hline la & 4 & 0 & $0 \%$ \\
Ib & 18 & 5 & $27,8 \%$ \\
Ila & 58 & 10 & $17,2 \%$ \\
Ilb & 8 & 4 & $50 \%$ \\
\hline Ilc & 38 & 4 & $10,5 \%$ \\
III & 20 & 2 & $10 \%$ \\
\hline TOTAL & 146 & 25 & $17,1 \%$ \\
\hline
\end{tabular}

De la totalidad de pacientes, el 76,7 \% fueron pacientes solo con EUP, mientras que $23,3 \%$, fueron pacientes con EUP y enfermedad renal crónica. De los 34 pacientes con enfermedad renal crónica, 22 estaban en estadio 5, ocho en estadio 4 y cuatro en estadio 3 . A pesar de que 22 pacientes estaban en estadio terminal, solo 18 de ellos 
estaban recibiendo hemodiálisis. El $52,1 \%$ de los pacientes tenían algún tipo de comorbilidad al momento del estudio. La comorbilidad más frecuente fue la hipertensión arterial, presente en el $28,8 \%$ de pacientes.

Como causa de la hemorragia digestiva, el $46,6 \%$ de los pacientes fueron por úlceras gástricas, mientras que el restante $53,4 \%$ por úlceras duodenales, que fue la causa predominante en ambos grupos de estudio, es decir, $51,8 \%$ en pacientes con enfermedad ulcerosa péptica sin enfermedad renal crónica y 58,8 \% en los pacientes con enfermedad ulcerosa péptica con enfermedad renal crónica $(p=0,47)$ (Figura 2$)$.

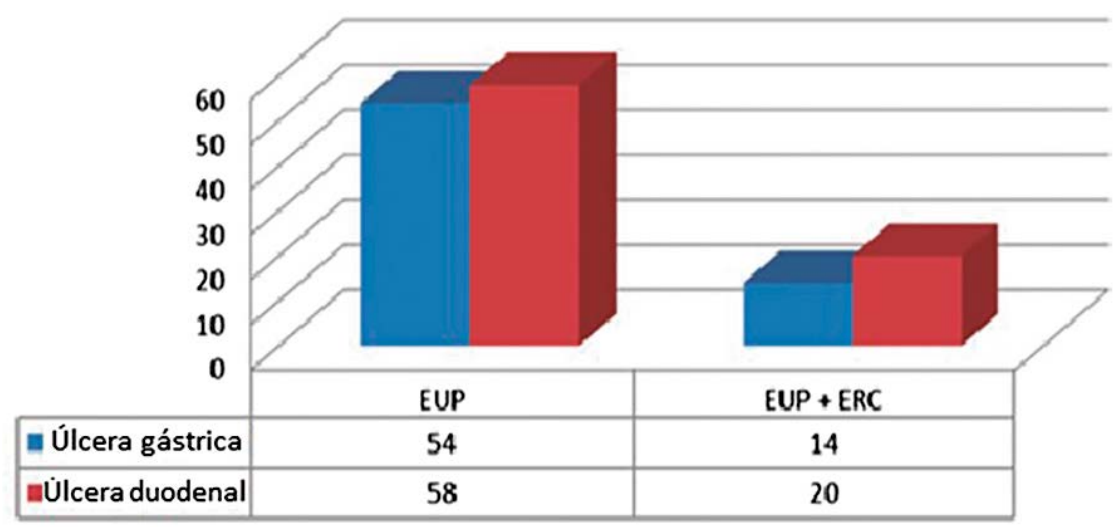

Figura 2. Distribución de la ubicación de la úlcera péptica de acuerdo al grupo de estudio $(p=0,47)$

La tasa de complicaciones en general para todos los pacientes fue del $21,2 \%$. Veinticinco pacientes $(17,1 \%)$ presentaron resangrado, dos pacientes tuvieron úlcera penetrada y 4 de ellos fallecieron. Cuando se analizaron las complicaciones en los dos grupos de estudio, se evidenció que en el grupo de pacientes con EUP sin ERC, once presentaron resangrado $(9,8 \%)$ dos fallecieron y las dos únicas ulceras penetradas estuvieron en este grupo de pacientes. Mientras que, de los 34 pacientes con EUP y ERC, 14 de ellos resangraron $(41,2 \%)$ y dos fallecieron. La diferencia fue significativa $(p<0,001)$. El odds ratio (OR) de resangrado en los pacientes con EUP con ERC fue de 6,43 con respecto a los pacientes con EUP sin ERC (Figura 3).

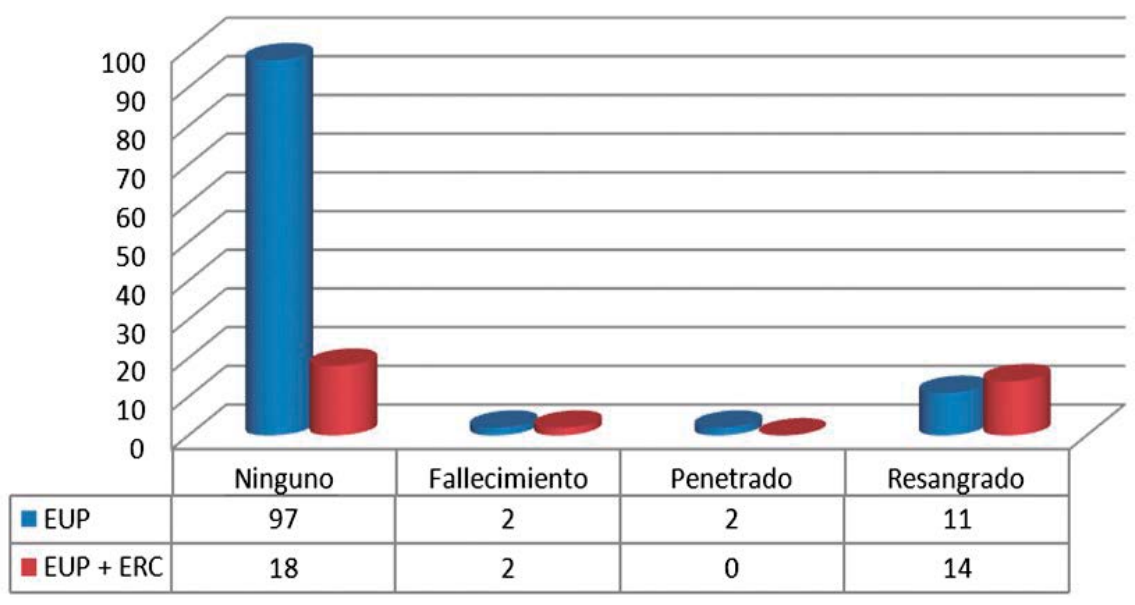

Figura 3. Distribución de las complicaciones de la úlcera péptica de acuerdo al grupo de estudio $(p<0,001)$ 
El 93,8\% de los pacientes salieron de alta después del episodio de sangrado digestivo, 5 pacientes fueron intervenidos quirúrgicamente y 4 pacientes fallecieron. De estos últimos, dos de ellos fallecieron por shock séptico y los 2 restantes con trastorno metabólico. De estos 5 pacientes sometidos a cirugía, en dos de ellos el motivo fue un vaso gigante no tratable endoscópicamente, mientras que los otros 3 fueron por persistencia de resangrado, de estos 5 pacientes sometidos a cirugía, 3 padecían de enfermedad renal crónica. $(p=0,057)$.

La tasa de mortalidad total para los pacientes con EUP sin ERC, fue de $1,7 \%$, mientras que la tasa de mortalidad de las úlceras pépticas en pacientes con enfermedad renal crónica fue del $5,9 \%$. $(p=0,2)$. En ambos grupos fallecieron 2 pacientes.

\section{DISCUSIÓN}

Las complicaciones de la enfermedad ulcerosa péptica son emergencias médicas comunes, las cuales contribuyen a una significativa morbilidad y mortalidad en todo el mundo. Se menciona que incluso el 5 al $10 \%$ de la población presentará alguna manifestación de enfermedad ulcerosa péptica a lo largo de su vida (1). Sin embargo, algunos estudios endoscópicos prospectivos muestran que cerca de la mitad de los pacientes con úlcera péptica son asintomáticos, por lo que podría esperarse que la verdadera prevalencia sea el doble de la anteriormente señalada, esta prevalencia es aún mayor en ciertas poblaciones de pacientes como son los usuarios de AINE u hospitalizados o en pacientes cirróticos ${ }^{(6,7)}$.

Actualmente existen reportes a nivel mundial que describen una disminución en la prevalencia de enfermedad ulcerosa péptica ${ }^{(8)}$.

Por otra parte, la enfermedad renal crónica es otra enfermedad de alta incidencia y prevalencia en nuestro medio. En un estudio realizado en el Perú, se encontró que la prevalencia de ERC en la población estudiada fue de $37 \%$, además se evidenció que la hipertensión arterial fue el antecedente más frecuente $(29,2 \%)$ seguido de diabetes mellitus tipo $2(16,3 \%)$ y ambas patologías $(11,31 \%)$. Solo $32,69 \%$ de pacientes no tuvieron antecedentes familiares ${ }^{(3,5)}$. Se sabe por algunas referencias bibliográficas que la enfermedad renal crónica es una patología que incrementa el riesgo de sangrado en los pacientes con úlcera péptica, siendo esta la mayor causa de hemorragia digestiva en los pacientes con enfermedad renal crónica. Se ha descrito que los pacientes con enfermedad renal crónica tienen mayor riesgo de resangrado de la úlcera péptica lo que acarrearía mayor cantidad de complicaciones en los pacientes nefrópatas ${ }^{(8)}$.

Usualmente, la enfermedad úlcera péptica tiene una prevalencia semejante en ambos sexos, sin embargo, en nuestro estudio el $74 \%$ de los pacientes fueron varones, lo cual da una razón varón/mujer de casi 3:1.
La edad media de presentación fue de 65,47 años, con un amplio margen, donde la edad mínima de presentación fue de 19 años y la máxima de 93 años. Estos resultados son muy semejantes a los encontrados en otros estudios como por ejemplo el de Parasa, el de Luo, el de Bang o el de Lin, (3,8-10), donde el promedio de edad de presentación siempre es entre la sexta y séptima década de la vida. Sin embargo, es importante destacar que, aunque la edad promedio era de 66 años, la gran mayoría de los pacientes, el $60 \%$ de los pacientes, tenían entre 70 y 90 años, lo cual nos da a entender que la edad avanzada es un factor importante a la hora de presentación de la hemorragia digestiva por enfermedad ulcerosa péptica.

Cuando se hizo un análisis demográfico comparativo entre los dos grupos de estudio, observamos que hay una diferencia significativa con respecto a la edad del paciente, el nivel de hemoglobina, el de plaquetas, evidentemente, la creatinina. Esto es importante para el riesgo de resangrado ya que los pacientes con enfermedad renal crónica tenían una edad mucho mayor que los pacientes con o sin nefropatía, esto podría influir en el riesgo mayor de resangrado y de mortalidad. Lo mismo ocurre con los niveles de hemoglobina y de plaquetas que son significativamente más bajos en el grupo con enfermedad renal, en nuestro estudio esto no tuvo implicancia con respecto a la mortalidad debido al bajo número de muertes registradas.

Las comorbilidades también son factores que pueden influir en el desenlace de la hemorragia digestiva de pacientes con enfermedad ulcero péptica, al margen de la enfermedad renal crónica, en nuestro estudio observamos que poco más de la mitad de los pacientes en estudio $(52,1 \%)$, tenían algún tipo de morbilidad asociada. La patología que más se ha asociado es la hipertensión arterial $(28,8 \%$ de los pacientes), esto puede ser explicado no solo porque la hipertensión arterial es una enfermedad prevalente en nuestro medio a partir de la segunda mitad de la vida, sino porque además es la patología que más se relaciona a la etiología de la enfermedad renal crónica, que es muchas veces, consecuencia de HTA.

Históricamente se conoce que la enfermedad ulcerosa péptica es más frecuente en el duodeno, en nuestro estudio, el $53,4 \%$ de los casos tuvieron una ubicación en el duodeno, y $46,6 \%$ de los casos fueron gástricas. Estos resultados difieren de otros estudios como por ejemplo el realizado en el año 2013, estudio realizado por Bang ${ }^{(8)}$, donde se encontró que la ubicación más frecuente de las úlceras, fueron gástricas, $66,7 \%$ y 33,3\% para las duodenales, esto es probablemente debido a la propia gastropatía urémica.

La razón de resangrado de la úlcera péptica en pacientes que padecen de enfermedad renal crónica, es 6,43 veces mayor que en los pacientes con enfermedad ulcerosa péptica que no padecían de enfermedad renal crónica. Esto es comparable con la mayoría de los estudios encontrados (11-15). 
La tasa de resangrado de acuerdo a la clasificación de Forrest, presenta datos que no suelen ser los habituales con respecto a la teoría, donde se conoce que la mayoría de resangrados se produce en las lesiones tipo Forrest la y lb, (16), sin embargo, en nuestro estudio, la mayor tasa de resangrado se observó en los pacientes con úlceras Forrest Ilb $(50 \%)$, seguido de las lesiones Forrest Ib con una tasa de $27,8 \%$. No encontramos lesiones Forrest la con resangrado, mientras que las Forrest III, tuvieron un $10 \%$ de resangrado. Estas diferencias pueden ser explicadas porque la lesión Forrest Ilb es una lesión ambigua que depende del tipo de terapia que se realice, en nuestro caso, todas las lesiones Forrest $\mathrm{llb}$, fueron sometidas a remoción del coágulo, se encontró sangrado activo en la mayoría de ellas, y se trataron endoscópicamente con inyectoterapia y un método de termocoagulación. Además, la escasa cantidad que se encontró con este tipo de lesión (8 pacientes) con 4 resangrados, tienen que ser tomados de manera cuidadosa y tener cuidado con la interpretación. Cuando se comparó con otros estudios, se vio que, por ejemplo, el estudio de Bang et al. ${ }^{(8)}$, mostró una tasa más alta de resangrado que la hallada en nuestro estudio (37,5\%). En el estudio de Lin et al. (10), el cual enroló a 101 pacientes, la tasa total de resangrado fue de $19 \%$. También en este estudio se mostró una tasa de resangrado mayor en los pacientes con enfermedad renal crónica terminal (43\%) y en los pacientes con falla renal crónica ( $21 \%)$, comparado con los pacientes con función renal normal en los que la tasa de resangrado solo fue de $12 \%$; diferencias que fueron estadísticamente significativas $(p=<0.001)$. En el estudio de Cheung et al. ${ }^{(12)}$, por su parte, encontraron que la tasa total de resangrado fue del 38,0 \% para los pacientes con enfermedad renal crónica terminal, mientras que los pacientes que solo tuvieron falla renal crónica tuvieron una tasa de resangrado del $14 \%$ y los pacientes con función renal normal del $12 \%$. La diferencia también fue significativa en estudio ( $p=0,002)$. Por consiguiente, la tasa de resangrado hallada en el nuestro estudio fue del $17 \%$.

Las úlceras duodenales fueron encontradas en estadios de mayor sangrando activo y de mayor riesgo de sangrado que la úlcera gástrica. En el estudio realizado por Nardone et al. (17), en el 2005, la mayoría de las úlceras encontradas fueron duodenales tanto en pacientes con falla renal crónica, como en pacientes con función renal normal, sin embargo, las erosiones gástricas fueron mucho más prevalentes que las duodenales en este estudio. Nosotros no tomamos en cuenta la presencia de solo erosiones, ya que tomamos en consideración pacientes con hemorragia digestiva alta por una ulcera manifiesta, esto debido a que las erosiones no son necesariamente una causa de hemorragia digestiva alta, y muchas veces son un diagnóstico subjetivo de acuerdo a la impresión del endoscopista que podría sesgar el estudio.

El 93,8 \% de nuestros pacientes salieron de alta luego del manejo y tratamiento de la hemorragia digestiva por úlcera péptica, el tiempo de hospitalización fue variable de acuerdo a las comorbilidades o descompensaciones asociadas. Cinco pacientes fueron sometidos a cirugía al no poder controlar el sangrado digestivo por técnicas endoscópicas habituales, los dos pacientes que fueron enviados a cirugía presentaban un vaso gigante en el lecho ulceroso con alto riesgo de sangrado incontrolable intraprocedimiento. Los otros 3 pacientes fueron sometidos a cirugía por resangrados persistentes. Otros 4 pacientes $(2,7 \%)$ fallecieron, dos de ellos fueron por trastornos metabólicos no asociados a hipovolemia, esto es debido a que el riñón es un principal regulador en el equilibrio ácido-básico del organismo, excretando hidrogeniones $(\mathrm{H}+)$ y reteniendo bicarbonato $(\mathrm{HCO}-)$ para mantener el equilibrio en situaciones como la alcalosis respiratoria o la acidosis respiratoria. Al no existir una adecuada excreción de $\mathrm{H}+$ y una producción disminuida de HCO3- se produce una alteración paulatina del equilibrio ácido-básico, ocasionando acidosis metabólica crónica. Los otros dos pacientes fallecieron por shock séptico. La tasa de mortalidad de la úlcera péptica en los pacientes que padecían de enfermedad renal crónica fue del 5,8 \%. En el estudio realizado por Bang et al. ${ }^{(8)}$, la mortalidad asociada a la hemorragia fue del $16,7 \%$, mientras que el en estudio de Parasa et al. (3), la tasa de mortalidad en los pacientes con falla renal crónica fue de $3 \%$ y cuando el paciente estaba en falla renal crónica terminal de $4,8 \%$.

Un dato que debe tomarse en cuenta es la mortalidad en cada grupo de estudio, fallecieron 2 pacientes en cada uno, pero es importante tomar proporcionalmente estos datos, ya que en el grupo que solo presentó enfermedad ulcerosa péptica, la tasa de mortalidad de $1,7 \%$, y en pacientes con enfermedad ulcerosa péptica y enfermedad renal crónica fue de $5,9 \%$. ( $(p=0,2)$; sin embargo, un dato para tomar en cuenta es el bajo número de muertes encontradas en cada grupo de estudio.

Se hizo un análisis de los pacientes de acuerdo a si estaban es estadio terminal (estadio 5) que estaban sometidos a hemodiálisis y aquellos que no lo estaban; y se evidenció que los que estaban sometidos a hemodiálisis tenía una tasa de resangrado ligeramente mayor que en el grupo que no lo estaba. Sin embargo, el análisis estadístico, reveló que no se encontró una diferencia significativa entre estos dos grupos formados, ni en resangrado ni en mortalidad. 18 de los 34 pacientes estaban sometidos a hemodiálisis.

Es importante mencionar que, de acuerdo a los resultados encontrados, la enfermedad renal crónica influye definitivamente en el pronóstico de un paciente con enfermedad ulcerosa péptica, una de las hipótesis que podría explicar esta mayor incidencia de complicaciones en los pacientes nefrópatas es la disfunción plaquetaria de origen urémico que se cree que es el factor más importante. En nuestro estudio el recuento de plaquetas tuvo una diferencia estadísticamente significativa entre los grupos estudiados, encontramos la cantidad de plaquetas más bajas en pacientes con enfermedad renal crónica, sin embargo, a pesar de este resultado, el conteo 
de plaquetas no incluye un estudio de la funcionalidad de estas. Otra teoría sobre la influencia de la enfermedad renal crónica en el pronóstico de las úlceras pépticas es la hiperacidez originada por hipergastrinemia, la cual puede estar relacionada con la severidad del compromiso renal, lo cual afectaría la integridad de la mucosa gástrica, y como consecuencia se produce una cicatrización inadecuada de las úlceras y la aparición de complicaciones posteriores. Es muy probable que otras comorbilidades puedan influir negativamente en los pacientes con nefropatía, sin embargo, en nuestro estudio estas variables no mostraron una influencia estadística en la tasa de complicaciones de los pacientes con úlcera péptica.

Como limitaciones de nuestro estudio, tenemos que considerar que no fue factible encontrar el dato adecuado del consumo de AINE y el tipo o tiempo en el cual estos estuvieron consumiendo dicho grupo de fármacos, lo cual podría considerarse como un factor que pueda involucrarse como causa de las hemorragias digestivas, al ser un estudio en parte retrospectivo, muchas veces estos datos están ausentes o no están registrados de manera adecuada.

En conclusión, la razón de resangrado de la úlcera péptica en pacientes que padecen de enfermedad renal crónica, es 6,43 veces mayor que en los pacientes con enfermedad ulcerosa péptica que no padecían de enfermedad renal crónica.

La tasa de resangrado de la enfermedad ulcerosa péptica en pacientes con enfermedad renal crónica, en la Unidad de Hemorragia Digestiva del Hospital Nacional Edgardo Rebagliati Martins fue del $41,1 \%$, mientras que en los pacientes sin enfermedad renal crónica fue de $9,8 \%$.

No se encontró diferencias estadísticamente significativas entre los pacientes con resangrado de úlcera péptica y hemodiálisis, frente a los que resangraron pero no estuvieron en hemodiálisis.

\section{REFERENCIAS BIBLIOGRÁFICAS}

1. Saad R, Scheiman J. Diagnosis and management of peptic ulcer disease. Clin Fam Pract. 2004;6(3): 569-87.

2. Goicoechea E, Chian A. Características clínico epidemiológicas de la enfermedad renal crónica en pacientes con hipertensión arterial y/o diabetes mellitus II. Hospital I Albrecht - Essalud, 2008. UCV. Scientia. 2010; 2(2); 61-72.

3. Parasa S, Navaneethan U, Sridhar AR, Venkatesh PG, Olden K. End-stage renal disease is associated with worse outcomes in hospitalized patients with peptic ulcer bleeding. Gastrointest Endosc. 2013; 77(4): 609-16.

4. KDIGO clinical practice guideline for the diagnosis, evaluation, prevention and treatment of chronic kidney disease-mineral and bone disorder (CKD-MBD). Kidney Int Suppl. 2009;(113):S1-130.

5. Levey AS, Atkins R, Coresh J, Cohen EP, Collins AJ, Eckardt $\mathrm{KU}$, et al. Chronic kidney disease as a global public health problem: approaches and initiatives - a position statement from Kidney Disease Improving Global Outcomes. Kidney Int. 2007; 72(3).247-59.

6. Montes P, Salazar S, Monge E. Epidemiological changes in peptic ulcer and their relation with Helicobacter pylori. Hospital Daniel A Carrion 2000-2005. Rev Gastroenterol Peru. 2007; 27(4):382-8.

7. Ichiyanagui C, Lozano R, Huamán C, Iparraguirre $H$. Enfermedad ulcerosa péptica en pacientes cirróticos. Rev Gastroenterol Peru. 1995; 15(1):15-9.

8. Bang CS, Lee YS, Lee $Y H$, Sung $H$, Park HJ, Kim HS, et al. Characteristics of nonvariceal upper gastrointestinal hemorrhage in patients with chronic kidney disease. World J Gastroenterol. 2013; 19(43): 7719-25.

9. Luo JC, Leu HB, Hou MC, Huang KW, Lin HC, Lee FY, et al. Nonpeptic ulcer, nonvariceal gastrointestinal bleeding in hemodialysis patients. Am J Med. 2013; 126(264):e25-e32.

10. Lin SC, Wu KL, Chiu KW, Lee CT, Chiu YC, Chou YP, et al. Risk factors influencing the outcome of peptic ulcer bleeding in end stage renal diseases after initial endoscopic haemostasis. Int J Clin Pract. 2012; 66(8):774-81.

11. Wu CY, Wu MS, Kuo KN, Wang CB, Chen YJ, Lin JT. Long-term peptic ulcer rebleeding risk estimation in patients undergoing haemodialysis: a 10-year nationwide cohort study. Gut. 2011; 60(8): 1038-42.

12. Cheung J, Yu A, LaBossiere J, Zhu Q, Fedorak RN. Peptic ulcer bleeding outcomes adversely affected by end-stage renal disease. Gastrointest Endosc. 2010; 71(1):44-9.

13. Luo JC, Leu HB, Huang KW, Huang CC, Hou MC, Lin HC, et al. Incidence of bleeding from gastroduodenal ulcers in patients with end-stage renal disease receiving hemodialysis. CMAJ. 2011; 183(18): 1345-51.

14. Boccardo P, Remuzzi G, Galbusera M. Platelet dysfunction in renal failure. Semin Thromb Hemost 2004; 30(5):579-89.

15. Rockall T, Logan R, Devlin H, Northfield T. Risk assessment after acute upper gastrointestinal haemorrhage. Gut. 1996; 38(3):316-21.

16. Holster IL, Kuipers EJ. Update on the endoscopic management of peptic ulcer bleeding. Curr Gastroenterol Rep. 2011; 13(6):525-31.

17. Nardone G, Rocco A, Fiorillo M, Del Pezzo M, Autiero G, Cuomo $R$, et al. Gastroduodenal lesions and Helicobacter pylori infection in dyspeptic patients with and without chronic renal failure. Helicobacter. 2005 10(1):53-8.

Fuentes de financiamiento:

Este artículo ha sido financiado por el autor.

Conflictos de interés:

El autor declara no tener ningún conflicto de interés

Correspondencia:

Edson Guzmán Calderón

Dirección: Prolongación Manco II 115, Torre A - 1101, San Miguel. Lima, Perú.

Teléfono: +51984767112

Correo electrónico: edson_guzman@hotmail.com

$\begin{array}{lcccc}\text { Recibido: } & 30 & \text { de mayo de } & 2018 \\ \text { Evaluado: } & 16 & \text { de junio de } & 2018 \\ \text { Aprobado: } & 12 & \text { de julio de } & 2018\end{array}$

(c) La revista. Publicado por Universidad de San Martín de Porres, Perú. (c) Br Licencia de Creative Commons Artículo en acceso abierto bajo términos de Licencia Creative Commons Atribución 4.0 Internacional. (http://creativecommons.org/licenses/by/4.0/)

ORCID iDs

Gerly Edson Guzmán Calderón

https://orcid.org/0000-0002-1673-3669 